ESJ Humanities

\title{
Espèces Ligneuses de Savanes Sèches du Nord du Togo : Considérations Socioculturelles et Relations de Pouvoir des Parties Prenantes
}

\author{
Badjaré Bériname \\ Doctorant, ir \& Master recherche, \\ Ecole supérieure d'agronomie, Université de Lomé, Togo \\ Adjayi Macomba Bétidé \\ Agronome, productions végétales, \\ Faculté des sciences de l'agriculture et de l'alimentation (FSAA), Université \\ Laval, Québec, Canada \\ Abbey Abbévi Georges \\ Maître de conférences, Département d'économie et sociologie rurales, \\ Ecole supérieure d'agronomie, Université de Lomé, Togo \\ Segla Kossi Novinyo \\ Docteur, Laboratoire de Recherche Forestière (LRF), \\ Faculté des Sciences, Université de Lomé, Togo
}

\section{Kokou Kouami}

Professeur titulaire, Laboratoire de Recherche Forestière (LRF),

Faculté des Sciences, Université de Lomé, Togo

\section{Bigou-Laré Nadédjo}

Professeur Agrégé, Laboratoire de recherche sur le management des organisations (LAREMO), Université de Lomé, Togo

\section{Doi:10.19044/esj.2021.v17n9p89}

Submitted: 23 January 2021

Accepted: 08 March 2021

Published: 31 March 2021
Copyright 2021 Author(s)

Under Creative Commons BY-NC-ND

4.0 OPEN ACCESS

Cite As:

Bériname B., Macomba Bétidé A., Abbévi Georges A., Kossi Novinyo S., Kouami K. \& Nadédjo B.L. (2021). Espèces Ligneuses de Savanes Sèches du Nord du Togo : Considérations Socioculturelles et Relations de Pouvoir des Parties Prenantes. European Scientific Journal, ESJ, 17(9), 89. https://doi.org/10.19044/esj.2021.v17n9p89

\section{Résumé}

Les espèces ligneuses du nord du Togo sont sujettes à de diverses utilisations pour satisfaire des besoins multiples des populations et leur gestion implique plusieurs acteurs. L'objectif de l'étude est d'apprécier les connaissances endogènes de ces espèces ligneuses, les préférences et les 
similarités. L'approche méthodologique est basée sur des enquêtes ethnobotaniques et socioéconomiques menées auprès de 14 groupes ethniques par interview individuel et par focus groups. Les analyses ont porté sur la Classification Ascendante Hiérarchique $(\mathrm{CAH})$ des usages selon la méthode de Sorensen et sur la catégorisation des parties prenantes. Au sein des différents groupes socioculturels ciblés, il ressort un consensus, notamment sur les espèces utilisées pour une catégorie d'usage donnée. L'utilisation courante des espèces ligneuses est orientée simultanément sur deux grands types d'usages à savoir : (i) usages alimentaires, médicinaux, fourragères et rituels ; et (ii) usages artisanal, bois énergie et bois de service. Elle est rare pour un type spécifique d'usages cosmétiques ou hygiéniques. Ces usages sont similaires à quatre grands regroupements ethniques. Le résultat des inventaires réalisé sur l'ensemble des espèces ligneuses montrent que, 42 de ces espèces présentent une vulnérabilité forte. Les réalités socioculturelles et la promiscuité entre les groupes ethniques déterminent les connaissances et les choix de biens et services écosystémiques. Ainsi, il est recommandé que les programmes d'aménagements forestiers tiennent compte de ces facteurs y compris, les responsabilités locales de même que les influences et les intérêts des parties prenantes.

Mots-clés : Espèces ligneuses, ; socioculturelles, ; Biens écosystémiques, U sages, Relations, Togo 


\title{
Woody Species of Dry Savannah in Northern Togo: Sociocultural Considerations and Power Relationships of Stakeholders
}

\author{
Badjaré Bériname \\ Doctorant, ir \& Master recherche, \\ Ecole supérieure d'agronomie, Université de Lomé, Togo \\ Adjayi Macomba Bétidé \\ Agronome, productions végétales, \\ Faculté des sciences de l'agriculture et de l'alimentation (FSAA), Université \\ Laval, Québec, Canada \\ Abbey Abbévi Georges \\ Maître de conférences, Département d'économie et sociologie rurales, \\ Ecole supérieure d'agronomie, Université de Lomé, Togo \\ Segla Kossi Novinyo \\ Docteur, Laboratoire de Recherche Forestière (LRF), \\ Faculté des Sciences, Université de Lomé, Togo \\ Kokou Kouami \\ Professeur titulaire, Laboratoire de Recherche Forestière (LRF), \\ Faculté des Sciences, Université de Lomé, Togo \\ Bigou-Laré Nadédjo \\ Professeur Agrégé, Laboratoire de recherche sur le management des \\ organisations (LAREMO), Université de Lomé, Togo
}

\begin{abstract}
Woody species in northern Togo are used to meet multiple needs of populations and their management involves many actors. The study objective is to appreciate communities' knowledge of these woody species, preferences, and similarities. The methodology is based on semi-directive ethnobotanical and socioeconomic surveys within 14 ethnic groups through individual interviews and focus groups discussions. The analysis included Hierarchical Upward Classification (HUC) of uses according to the Sorensen method, and on the characterization of the main stakeholders. Within the targeted various socio-cultural groups, there is a consensus, mainly on the species used for a given category of use. It emerges that woody species are frequently used for two goals (food and medicinal; livestock feeding and ritual), or three goals (artisanal, energy wood, and service wood) and rarely for a specific type of use (cosmetic or hygienic). Four main ethnic groups have similarities of uses and 42 out of all of the species identified, present a high vulnerability. Some local socio-cultural realities and promiscuity between ethnic groups determine
\end{abstract}


the knowledge of forest trees and the choices of goods and services. Therefore, it is recommended for forest development programs to take into account the above factors including local responsibilities as well as influences and interests of stakeholders.

Keywords: Woody species, Sociocultural, Ecosystem goods, Uses, Relationship, Togo

\section{Introduction}

Considérés comme des biens en commun (Yelkouni, 2012), les espèces ligneuses sont pourvoyeuses de services nécessaires à la société et en particulier à l'économie des ménages (Loubelo, 2012). Au regard des besoins variables des utilisateurs, l'un des enjeux majeurs reste le manque de connaissances spécifiques des attributs des multiples produits issus de ces espèces ligneuses (Guillemette, 2014). L'initiative internationale sur l'évaluation des écosystèmes pour le millénaire relève que chaque être humain dépend des « services écologiques » et il existe une vision économique des relations entre les hommes et leur environnement (MEA, 2005). Effectivement, les communautés locales récoltent directement, en général, de nombreux produits forestiers (Kokou et al., 2006b; Sop et al., 2010) confirmant le rôle crucial de la forêt dans le développement socio-économique et les réponses aux besoins des populations. Des études ont abordé la problématique des plantes utiles soit sur un type précis d'usage (Wala, 2001 ; Kokou et al., 2009 ; Batawila et al., 2010 ; Zabouh, 2014) soit sur une espèce spécifique (Dourma, 2008 ; Atakpama et al., 2014 ; Kebenzikato et al., 2014) soit encore sur la valeur socioculturelle des plantes alimentaires mineures dans un contexte de préservation (Batawila et al., 2010; Akpavi et al., 2013). Cependant, sur le continent africain, des préoccupations persistent sur la résilience des systèmes sociaux et environnementaux ainsi que sur le taux élevé de déforestation (FAO, 2012) et la gestion durable du milieu naturel.

En réponse, des programmes d'aménagement ou de restauration initiés ont montré des limites, notamment sur la suffisance prise en compte des considérations sociales, relatives à l'utilité de certaines espèces ligneuses et aux préférences des populations qui attachent des valeurs à ces ressources locales (Ouédraogo et al., 2014 ; Traoré et al., 2011 ; Sop et al., 2010 ; Taïta, 2003 ; Sop et al., 2010 ; Traoré et al., 2011 ; Ouédraogo et al., 2014). Sur les fonctions et usages des espèces ligneuses, Lykke, (1998) ; Hahn-Hadjali et al., (2003) Lykke et al., (2004) ; Hahn-Hadjali et al., (2003) puis Lykke, (1998) Lykke et al., (2004).

Au Togo, l'augmentation des populations, a entraîné une pression sans cesse croissante sur les écosystèmes forestiers due essentiellement aux activités anthropiques de tout genre (feux de brousse, exploitations 
anarchiques de certaines essences recherchées pour le charbon ou pour le bois de feu et pour le bois d'œuvre, divagations des animaux, pratiques culturales traditionnelles, etc.). Celles-ci ont conduit à la raréfaction de l'herbe et au dépérissement des espèces ligneu ses. La régression des espèces ligneuses est plus prononcée pour les espèces fourragères, celles qui entrent dans les activités économiques, socioculturelles et celles qui occupent des places de choix dans la satisfaction des besoins de santé des hommes ( Touré, 2001 ; Segla et al., 2015,). Face à cette situation il s'avère nécessaire d'entreprendre des études devant fournir des informations sur les fonctions et usages des biens et services issus d'espèces ligneuses, les considérations socioéconomiques et les relations de pouvoirs des parties prenantes. En effet, le manque d'informations scientifiques, en particulier sur les fonctions et usages des biens et services issus d'espèces ligneuses, les considérations socioéconomiques et ethniques et les relations de pouvoirs des parties prenantes sont sans doute parmi les contraintes de gestion et de valorisation des ressources forestières (Zohoun et al., 2002 ; Brahic et Terreaux, 2009a ; Brahic et Terreaux, 2009b ; Kébézikato, 2014). Par ailleurs, certains chercheurs pensent que la gestion des biens éco systémiques passe par la définition des organismes qui produisent les services (Kremen et Osfeld, 2005) et une meilleure compréhension des dynamiques spatiotemporelles dans l'approvisionnement de ces services (Luck et al., 2009). Aussi, face aux contradictions des intérêts des acteurs, l'analyse de leurs pouvoirs s'avère telle utile pour l'aide à la décision et l'amélioration des stratégies et des moyens de subsistance (Mayers, 2005).

L'objectif général de cette étude est de contribuer à la meilleur valorisation des espèces ligneuses utiles des savanes soudaniennes du Nord du Togo. De manière spécifique, il s'agit (i) d'analyser les fonctions et les usages des espèces ligneuses utiles connus par divers groupes ethniques dans la $\mathrm{s}$ avane sèche du Nord du Togo ; ; (ii) analyser les responsabilités et les intérêts des parties prenantes.

\section{Matériel et Méthodes}

\subsection{Zone d'étude}

L'étude a été réalisée au nord du Togo dans les zones écologiques I et II cor respondant à la s avane sèche ( Ern, 1979) et composée de trois régions économiques (Savanes, Kara et Centrale) couvrant un peu plus de $40 \%$ du territoire togolais (Figure 1). Au total, quatorze principaux groupes ethniques se répartissent sur cette zone avec d'une part, les Moba, les Gourmantché, les Tchokossi, les Gam-gam et les Yenga que l'on retrouve dans les préfectures de Tône, de Tandjoare, de Kpendjal, de l'Oti et de Cinkassé et d'autre part, les Bassar, les konkonba, les Naouda, les Kabyè, les Lamba, les Solla, les Lougba, les Temberma et les Tem qui peuplent les 
préfectures de Bassar, de Dankpen, de Doufelgou, de la Kozah, de la Binah, de la Kéran et d'Assoli. En plus de ces groupes ethniques, on note dans la zone, la présence d'autres ethnies à savoir les Mossi, les Peuls et les Natchanba (Gayibor, 1997).

Sur le plan socioéconomique diverses activités relevant essentiellement du secteur agricole sont pratiquées par les populations pour tirer des revenus divers. En outre, certaines essences forestières associées aux cultures annuelles à travers des pratiques d'agroforesteries et d'autres, exploitées au sein des peuplements forestiers ou de boisements en zone rurale, génèrent des revenus non négligeables pour des populations (Agboh et Badjaré, 2007).

En général, il s'agit des produits forestiers ligneux issus des espèces à multiusage, principalement, Vitelaria paradoxa (les noms scientifiques des espèces doivent être en Italic), Andansonia digitata, Parkia biglobosa, Borassus aethiopium (Aleza et al., 2014 ; Kebenzikato et al., 2014). Dans la sSavane $\mathrm{s}$ sèche, il existe des aires protégées et des domaines du peuplement végétal constitués de boisements librement exploités par des particuliers. Selon la Loi $\mathrm{N}^{\circ} 2008-009$ du 19 juin 2008 portant le code forestier, sont assimilés aux particuliers, les personnes physiques ou morales, les groupements ou communautés rurales ou de base qui n'entrent pas dans la catégorie des collectivités territoriales. 


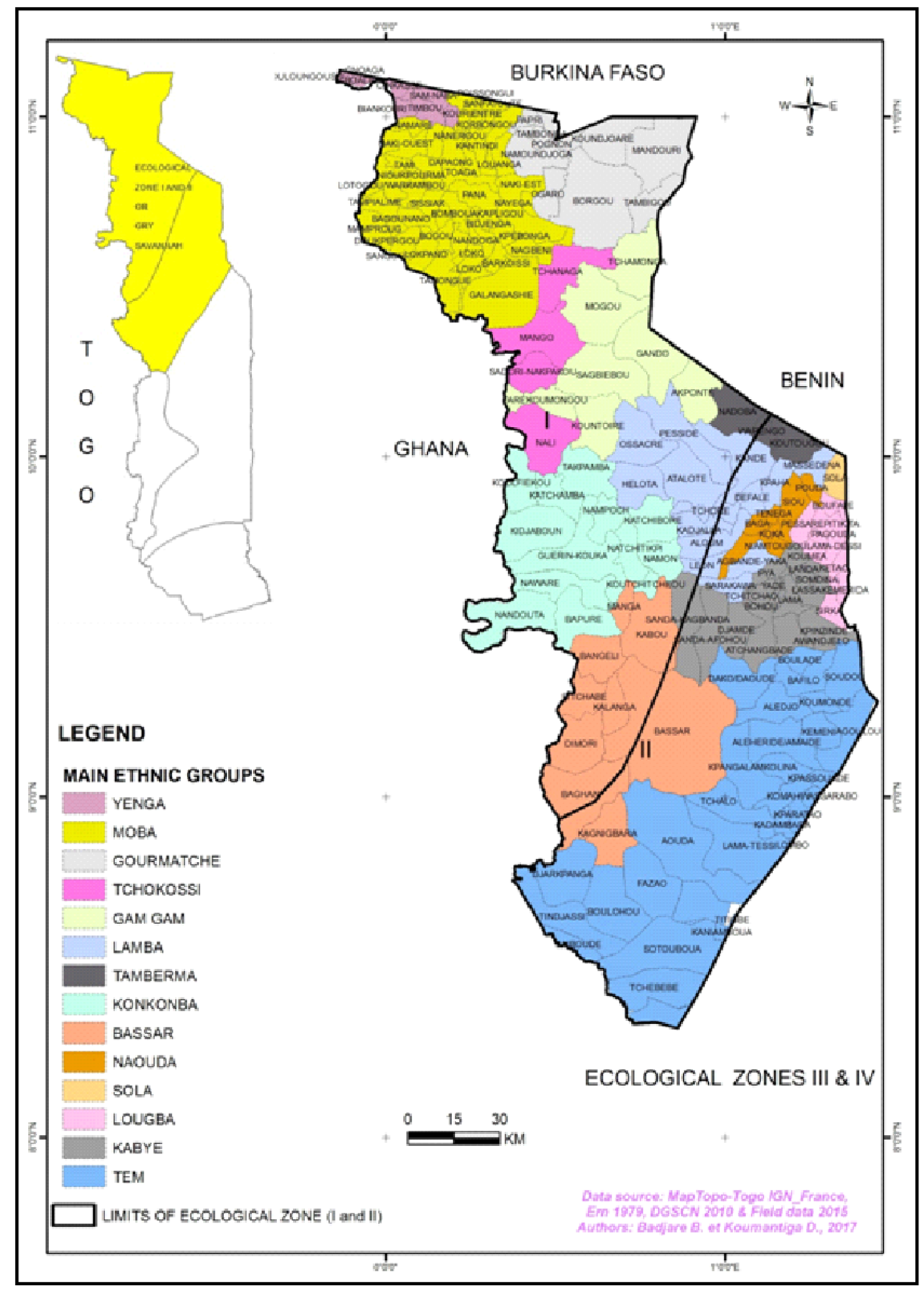

Figure 1 : Zone de l'étude et de distribution des groupes ethniques enquêtés

\subsection{Méthodes de collecte des données}

\subsubsection{Echantillonnage et source de données}

L'échantillonnage stratifié (Atakpama et al., 2012 ; Zabouh, 2014 ; ) a été adopté dans cette étude avec une distinction de trois strates (deux (02) régions cibles, notamment, Savanes et la Kara ; 12 préfectures des deux régions citées et 36 cantons au sein desquels, des villages sont ciblés pour la conduite des enquêtes). Il a été adopté une méthodologie d'enquêtes 
ethnobotaniques semi-directives par interview individuel et discussions en focus groups avec les groupes ethniques Moba, Yenga, Gourmantché, Gam Gam, Tchokossi, Lamba, Naouda, Kabyè, Bassar, Konkonba, Solla, Temberma et Tem. Les participants aux focus groups sont essentiellement les populations locales relevant de divers groupes socioprofessionnels, les leaders communautaires, les jeunes hommes et femmes, ... L'analyse des usages et des regroupements similarités au sein des groupes ethniques a porté sur les espèces répertoriées dans l'ensemble de la zone par Badjaré et al (2018). Les responsabilités des acteurs, parties prenantes sont analysées en référence aux indications du code forestier (Loi $\mathrm{N}^{\circ} 2008-009$ du 19 juin 2008) et des observations pratiques sur le terrain.

\subsubsection{Profil socioéconomique (profil socioéconomique)}

Les personnes enquêtées appartiennent à quatorze groupes ethniques que sont les Moba, les Gam Gam, les Gourmantché, Tchokossi, les Kabyè, les Lamba, les Konkomba, les Lougba, les Naouda, les Tem, les Bassar, les Solla, les Temberma et les Yenga. Elles sont âgées d'au moins 20 ans, composées donc de jeunes, d'adultes et de personnes de $3^{\text {ème }}$ âge (pourquoi exclure les personnes de $3^{\text {ème }}$ âges ??? car ces derniers sont garants d'une gestion durable), vivant dans les zones ciblées et au nombre de 558 dont 41,6\% de femmes et $58,4 \%$ d'hommes. La répartition en proportion des enquêtés par zone enquêtée est présentée sur la figure 2 .

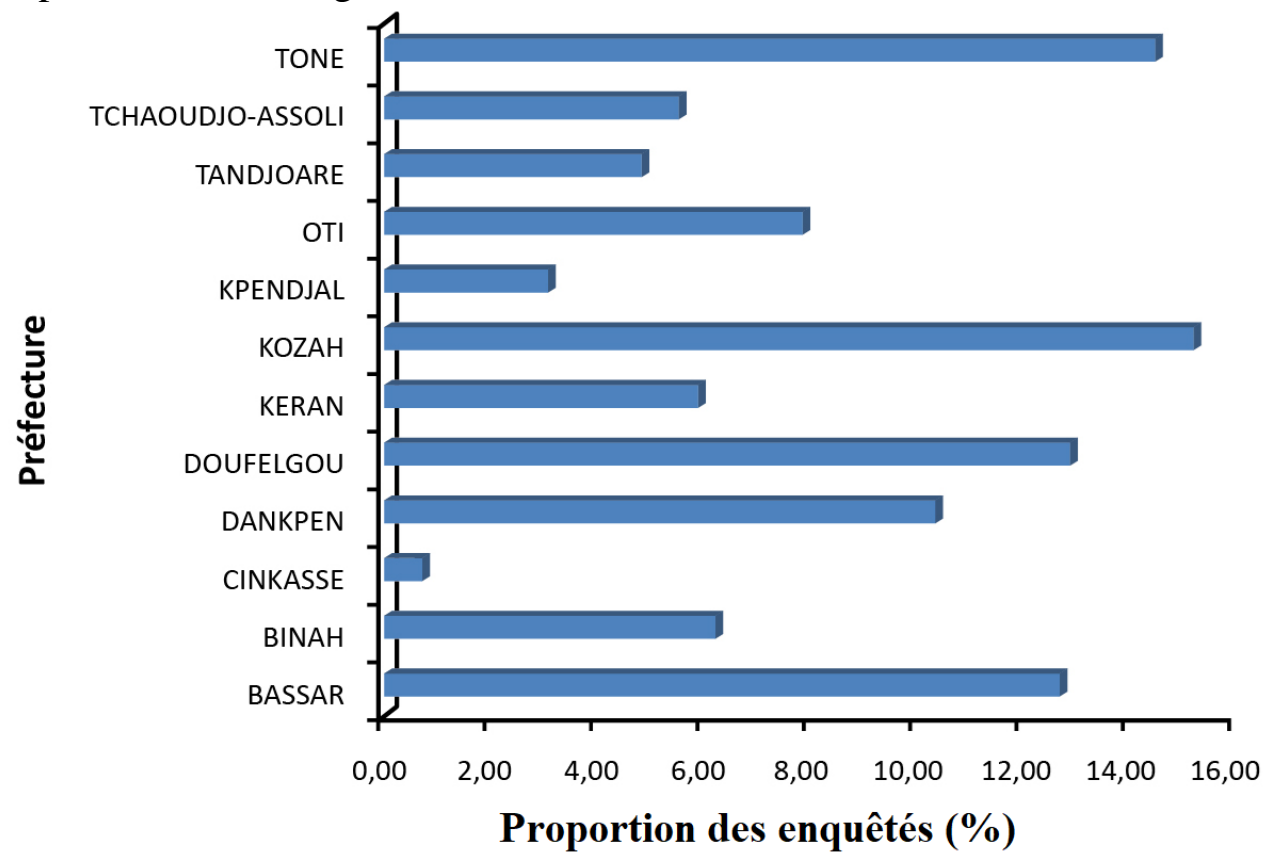

Figure 1: Répartition de la population enquêtée par préfecture 


\subsubsection{Enquêtes et indicateurs d'analyse}

Ciblant des populations riveraines de peuplements forestiers dans les régions des Savanes, de la Kara et Centrale, la collecte des données a permis de rassembler les informations sur : (i) les usages possibles des espèces ligneuses ainsi que des biens et services écosystémiques issus de ces ressources, (ii) les connaissances locales des valeurs de ces actifs naturels et des biens rattachés (iii) l'importance socioéconomique et les préférences des populations suivant les zones et les groupes ethniques. Les enquêtes ethnobotaniques ont intégré la collecte de données sociodémographiques. Les informations sur les valeurs traditionnelles ou coutumières des espèces ainsi que l'utilité ou l'utilisation qui est faite de chaque arbre forestier dans son milieu, notamment l'utilité des feuilles, des fruits, des graines, de l'écorse, du bois, etc ont été également collectées.

Par rapport aux principauxles parties prenantesacteurs, sont identifiées : (i) les propriétaires terriens/fonciers, (ii) les éleveurs transhumants, (iii) la communauté d'usagers de biens et services éco systémiques, les producteurs de bois énergie, (iv) la chefferie traditionnelle, (v) les organismes publics ou gouvernementaux, (vi) les chercheurs et spécialistes et (vii) les organisations non gouvernementales (ONG). Les rôles et responsabilités de ces différents acteurs ainsi que les relations d'interdépendance ont été également analysées à travers les entretiens.

\subsection{Traitement des données}

\subsubsection{Méthode de regroupements d'usages et d'analyse de similarités suivant les groupes ethniques}

Les traitements ont consisté en une analyse multivariée dont la classification ascendante hiérarchique $(\mathrm{CAH})$ des usages selon la méthode de Sorensen grâce au logiciel Community Analysis Package (CAP). Cette démarche d'analyse de gradient utilisée dans les analyses de correspondance canonique directe (Canonical Correspondance Analysis (CCA)) basée sur le "Whittaker's Index of Association (WIA)" permet d'ajuster la similarité des résultats. Pour ce faire, deux matrices ont été créées sur tableur Excel, sur la base de l'abondance (présence signalée des espèces sur les terroirs) et de la dominance (importance en termes de recours par les populations) des différentes espèces recensées dans les zones cibles. La première matrice « espèces-catégorie d'usage » soumise au logiciel CAP a permis de ressortir le dendrogramme présentant les similarités suivant les grandes catégories d'usages. La seconde matrice " espèces-ethnie » soumise au même outil a permis de ressortir le dendrogramme présentant les similarités suivant les différents groupes ethniques. Au regard du niveau de significativité, les regroupements ont été discriminés. L'analyse de la diversité et des similarités d'usages sont conduites sur base de recours aux biens et services éco 
systémiques dépendant des connaissances locales des utilisateurs. Une espèce d'arbre forestier est qualifiée de dominante dans un milieu pour un groupe humain donné lorsque la distribution de fréquences spécifiques liées aux utilisations citées (fréquences de citation) par les enquêtés, montre une fréquence relative de 1 ' espèce supérieure ou égale à $25 \%$.

La fréquence de citation (F) d'une espèce correspond au rapport entre le nombre d'enquêtés (n) ayant cité l'espèce et le nombre total d'enquêtés $(\mathrm{N})$.

\subsubsection{Analyse des responsabilités et intérêts des parties prenantes}

A partir de la caractérisation des parties prenantes identifiées, notamment les propriétaires terriens, les éleveurs transhumants, la communauté d'usagers de biens et services éco systémiques, les producteurs de bois énergie, la chefferie traditionnelle, les organismes publics ou gouvernementaux et les organisations non gouvernementales (ONG), les analyses ont circonscrit les descriptions de rôles, de pouvoirs et des influences et exploré les indications de la Loi $\mathrm{N}^{\circ} 2008-009$ du 19 juin 2008 portant code forestier du Togo. Il est ainsi abordé les responsabilités, les potentiels et les influences des différents acteurs, les coalitions éventuelles envisageables dans les relations de pouvoirs, les intérêts et les avantages réciproques y compris les conflits éventuels. Dans la démarche, il est spécifiquement mis en évidence pour les communautés d'usagers de biens, les caractéristiques liées au sexe, à l'âge, au niveau d'instruction, à la profession et au groupe ethnique.

\section{Résultats}

\subsection{Usages des espèces ligneuses dans la $\mathrm{s}$ avane sèche au Nord du Togo}

Dans la $\mathrm{s}$ avane sèche, il est identifié et reconnu par les populations pour ce qui est des espèces ligneuses, les usages : (i) alimentaire, (ii) médicinal ou pharmaceutique, (iii) artisanal, (iv) bois énergie, (v) bois de service, (vi) fourragère, (vii) rituel, (viii) hygiéniques et (ix) cosmétique. L'ensemble des citations enregistrées (4.021) donnent comme fréquence, $34,0 \%$ pour l'utilisation alimentaire, $15,4 \%$ pour les besoins pharmaceutiques ou médicinaux, $12,2 \%$ pour les besoins en bois-énergie, $10,0 \%$ pour l'utilisation fourragère, $7,4 \%$ pour la destination artisanale, $6,8 \%$ pour l'hygiène corporelle, $6,7 \%$ pour le bois de service, $4,6 \%$ pour les besoins rituels ou magico-spirituels et $2,9 \%$ pour les réponses aux besoins cosmétiques.

Les résultats montrent que ces usages portent essentiellement sur les feuilles, les racines, les fruits, les graines, les fleurs, le bois et les écorces. Les déterminations de valeurs d'usage tiennent compte des différentes sollicitations d'organes d'espèces ligneuses dont les destinations varient selon les usages connus des populations ciblées. Les fréquences de citations sur l'utilité des organes sont respectivement de $32,9 \%$ pour les fruits, $26,7 \%$ pour 
les feuilles, $12,6 \%$ pour le bois, $11,7 \%$ pour les graines, $7,4 \%$ pour les écorces, $7 \%$ pour les racines et $1,7 \%$ pour les fleurs (figure 2 ). Les fruits sont donc les plus utilisés dans la zone suivis respectivement des feuilles, du bois, des graines, des écorces, des racines et des fleurs.

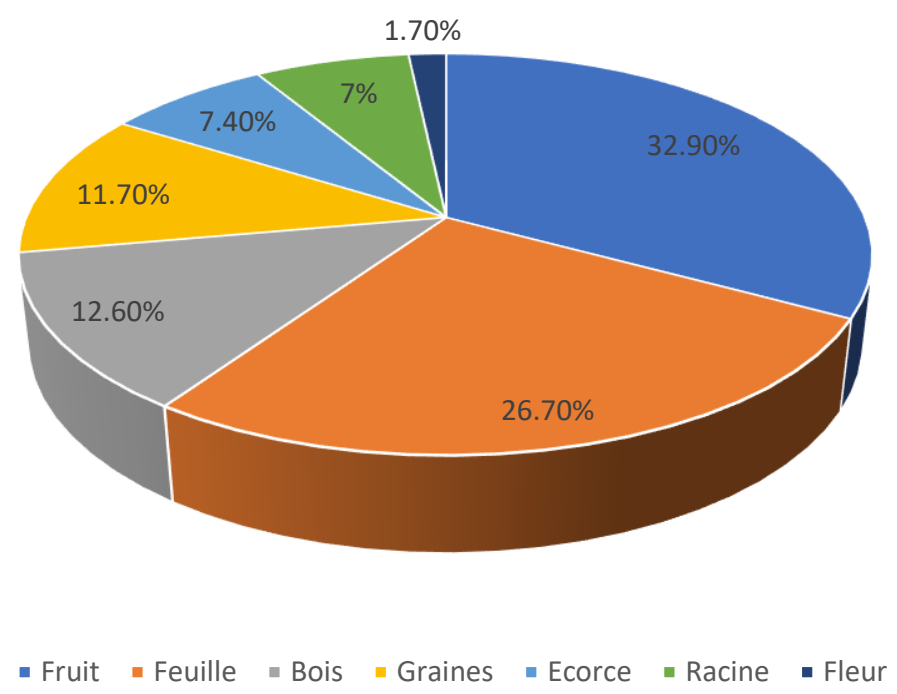

Figure 2 : Fréquence de citation des organes d'espèces ligneuses de savane sèche (Nord Togo)

\subsection{Regroupements des usages d'espèces ligneuses et similarités suivant les groupes ethnoculturels}

2.1.1. Catégories d'usages d'espèces ligneuses au nord du Togo

A partir de l'ajsutement réalisé par la méthode'’Whittaker's Index of Association' ' basée sur l'analyse de gradient, il ressort de la catégorisation des usages d'espèces répértoriées, des indications exploitables contribuant à la réflexion. Dans un intervalle de significativité de 50 à $75 \%$, les espèces ligneuses exploitées par les communautés ciblées dans la $s$ avane sèche sont généralement utilisées à la fois pour deux ou trois usages et rarement pour un type spécifique d'usage. Ainsi, comme l'illustre la figure (figure 3), le groupe 1 (GU1) présente la combinaison des catégories d'usages " alimentaire et médicinal » tirés de la même espèce d'arbre forestier. Le groupe d'usage 2 (GU2) qui se compose de deux sous-groupes (GU2a et GU2b) présente pour le sous-groupe GU2a, le regroupement des usages « artisanal-bois de service et bois énergie » et le regroupement «fouragère et rituel » pour le sousgroupe GU2b. Seuls les catégories d'usages hygiènique (GU3) et cosmétique (GU4) se rapportent à des espèces spéficiques exploitées uniquement pour des besoins liés à ces types d'usages. Pour ces deux dernières catégories, les 
espèces d'arbres sollicités sont exclusivement utiles à ces usages dans un intervalle de significativité de 70 à $75 \%$ (figure 3 ).

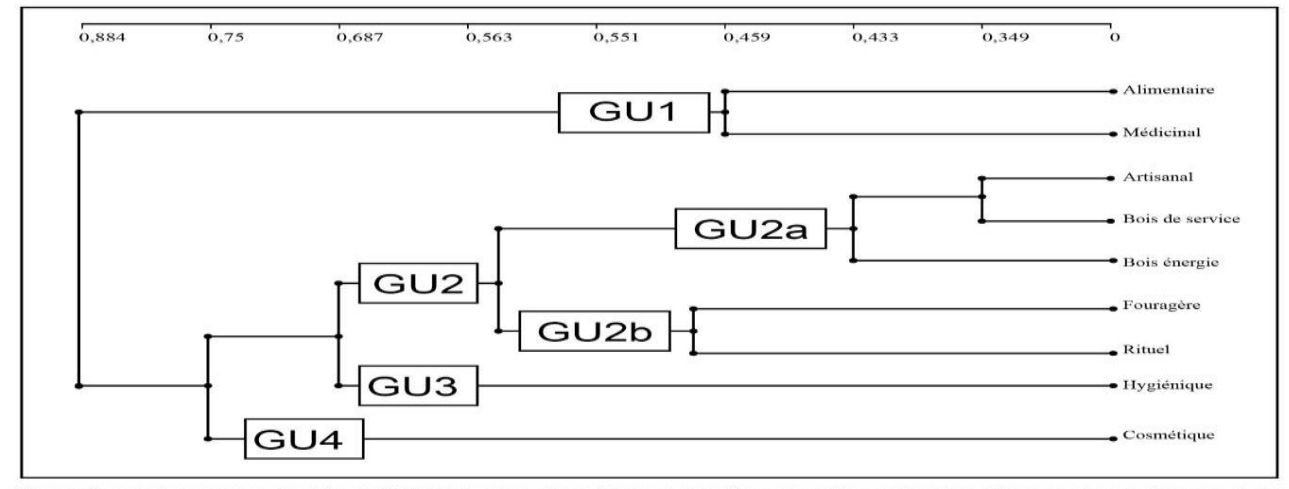

Figure 3 : Regroupements des usages des espèces ligneuses dans la s avane sèche

\subsubsection{Similarités au sein des groupes ethnoculturels}

La distribution spatiale des espèces ligneuses utiles au sein des groupes ethnoculturels interrogés dans la $\mathrm{s}$ avane sèche au nord Togo, montre que les ethnies Tem, Konkonba, Moba, Kabyè, Bassar, Naouda, Lamba et Tchokossi ont relevé le plus grand nombre d'espèces dépassant $35 \%$ du nombre total (149) répertorié avec respectivement, 67, 63, 62, 62, 60, 56, 54 et 53 espèces citées. L'ajustement sophistiqué des résultats du CCA par la méthode WAI basée sur l'abondance des espèces ligneuses dans chaque groupe ethnoculturel permet d'obtenir un classement en quatre groupes (G1, G2, G3 et G4) dont deux sous-groupes de G2 (G2a et G2b) comme l'indique la figure 4.

La répartition spatiale des différentes ethnies dans la s avane sèche au nord Togo montre que les différents groupes constitués (figure 4) se retrouvent en général sur le même espace géographique (Legendre et al., 2005). Certaines espèces ligneuses sont relativement et spécifiquement connues par des groupes ethniques donnés. C'est le cas par exemple d'espèces d'arbres qui ne sont pas utilisées par les groupes ethniques, Konkonba, Lamba, Naouda et Moba.

$\mathrm{Au}$ sein du groupe Bassar-Konkonba-Tem (G1), sur 92 espèces ligneuses citées, $45,65 \%$ sont connues à la fois par les trois ethnies et $30,43 \%$ par au moins deux ethnies du groupe constitué. Les espèces notoirement connues par l'ensemble du groupe Bassar-Konkonba-Tem sont rangées au sein de 22 familles. Les plus représentatives sont :

Les plus représentatives sont: Fabaceae (Acacia albida, Parkia biglobosa, Prosopis Africana, Afzelia Africana, Daniellia oliveri, Isoberlinia doka), Arecaceae (Borassus aethiopum, Hyphaene thebaica, Elaeis guineensis), Meliaceae (Azadirachta indica, Pseudocedrela kotschyi, Khaya 
senegalensis), et Malvaceae (Bombax costatum, Adansonia digitata, Ceiba pentandra).

$\mathrm{Au}$ niveau du groupe Gourmantché-Tamberma-Yenga (G2a), 70 espèces ligneuses sont citées dont $37,14 \%$ sont connues des groupes ethniques et $22,86 \%$ par deux ethnies du groupe. Les espèces communes à l'ensemble du groupe et citées par chacune des ethnies se répartissent dans 15 familles qui sont principalement Fabaceae (Afzelia africana, Parkia biglobosa, Prosopis africana, Detarium microcarpum, Tamarindus indica, Senna siamea), Anacardiaceae (Lannea microcarpa, Lannea barteri, Anacardium occidentale, Mangifera indica), Meliaceae (Pseudocedrela kotschyi, Khaya senegalensis, Azadirachta indica), Malvaceae (Bombax costatum, Ceiba pentandra, Adansonia digitata), , Verbenaceae (Vitex doniana, Tectona grandis) et Sapotaceae (Vitellaria paradoxa).

Le groupe Kabyè-Lamba (G2b) a cité au total 76 espèces ligneuses et $61,84 \%$ sont connues des deux ethnies contre $38,16 \%$ connues par seulement l'une des ethnies. Les espèces connues par l'ensemble du groupe sont identifiées au sein de 9 principales familles qui sont

Annonaceae (Annona ondulata, Annona senegalensis), Moraceae (Antiaris africana, Ficus thonningii, Ficus sur, Ficus polita, Milicia excelsa), Fabaceae (Tamarindus indica, Senna siamea, Afzelia africana, Burkea africana, Detarium microcarpum, Daniellia oliveri, Parkia biglobosa, Prosopis africana, Leucaena leucocephala), Rutaceae (Afraegle paniculata, Citrus limon, Citrus sinensis), Malvaceae (Bombax costatum, Adansonia digitata, Ceiba pentandra), Arecaceae (Borassus aethiopum, Elaeis guineensis, Hyphaene thebaica), Verbenaceae (Vitex doniana, Tectona grandis, Gmelina arborea), Sapindaceae (Blighia sapida) et Anacardiaceae (Anacardium occidentale, Mangifera indica).

De l'analyse des citations du groupe G3 composé des ethnies Gam Gam-Moba-Tchokossi, il ressort qu'au total 82 espèces ligneuses sont connues de ce groupe localisé à l'extrême nord du pays. Près de $35 \%$ de ces arbres sont cités par chacune des ethnies et environ $10 \%$ sont citées par deux ethnies du groupe sur les trois. Les espèces communes au groupe sont rangées au sein de 11 familles que sont, Combretaceae (Pteleopsis suberosa), Malvaceae (Adansonia digitata, Grewia lasiodiscus, Ceiba pentandra Bombax costatum), Arecaceae (Cocos nucifera, Elaeis guineensis, Borassus aethiopum), Zygophyllaceae (Balanites aegyptiaca), Anacardiaceae (Lannea microcarpa, Anacardium occidentale, Mangifera indica), Fabaceae (Parkia biglobosa, Tamarindus indica, Detarium microcarpum, Prosopis africana, Faidherbia albida), ), Rubiaceae (Gardenia erubescens), Rutaceae (Citrus sinensis), Moringaceae (Moringa oleifera), Ebenaceae (Diospyros mespiliformis), Verbenaceae (Vitex doniana) et Sapotaceae (Vitellaria paradoxa). 
Pour le groupe Lougba-Naouda-Solla (G4), il se dégage 54 espèces ligneuses utiles connues dont près de $28 \%$ par chacune des trois ethnies et environ $22 \%$ par au moins deux ethnies du groupe. Les espèces communes à ce groupe ethnique sont organisées au sein de 6 familles et les principales sont Fabaceae (Daniellia oliveri, Prosopis africana, Parkia biglobosa, Leuceana leucocephala, Senna siamea, Afzelia africana), Malvaceae (Bombax costatum, Adansonia digitata, Ceiba pentandra), Arecaceae (Hyphaene thebaica, Borassus aethiopum, Elaeis guineensis), Meliaceae (Azadirachta indica, Khaya senegalensis, Pseudocedrila kotschyi), Verbenaceae (Gmelina arborea) et Anacardiacaea (Mangifera indica, Lannea microcarpa, Lannea Barteri, Lannea acida).

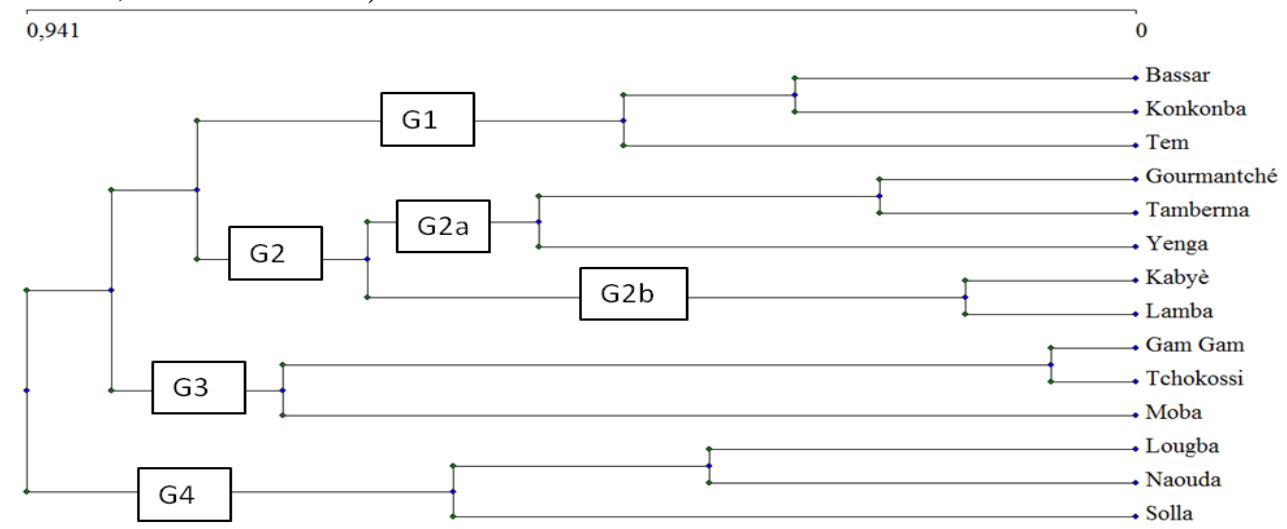

Figure 4 : Liens et similarités entre les groupes ethniques sur l'utilisation des espèces ligneuses de savane sèche

\subsection{Responsabilités et intérêts des parties prenantes}

Les propriétaires terriens/fonciers : Généralement autochtones, membres de collectivités et en plus grand nombre, les propriétaires fonciers sont responsables de domaines forestiers des particuliers (Titre III, chapitre $1^{\mathrm{er}}$, section $3 \mathrm{du}$ code forestier du Togo). Très importants, ils jouissent d'une influence et disposent d'un potentiel utile à la gestion durable de ces ressources. Ils sont souvent en conflit avec les éleveurs transhumants qui conduisent à leur gré et sans aucune concertation, les animaux sur les espaces naturels ou boisés et sur lesquels le bétail prélève des organes d'espèces ligneuses utiles. Aussi, les textes en vigueur confèrent-ils le droit à tout citoyen, propriétaire foncier ou non de planter des arbres mais exigent l'autorisation préalable des services compétents avant tout abattage.

Les éleveurs transhumants : Dans la s avane sèche, des conflits sont assez récurrents entre éleveurs transhumants et propriétaires fonciers, en général du fait de la responsabilité présumée des premiers sur la destruction par les animaux, des cultures et autres espaces boisés des derniers. Le mode d'élevage pratiqué par 1 es éleveurs sans respect des couloirs définis à cet 
effet, détruit les moyens d'existence des communautés, notamment les biens et services éco systémiques d'espèces ligneuses.

La communauté d'usagers de biens et services éco systémiques: Se comportant généralement comme des bénéficiaires d'actifs naturels, les membres des communautés locales y tirent régulièrement entre autres, des aliments, des produits pour la pharmacopée et du bois pour les services et l'énergie. Activement impliqués dans l'exploitation, la régénération et la gestion des ressources d'espèces ligneuses, ces acteurs de base ont des besoins et intérêts qui affectent directement la durabilité du milieu naturel. La législation en vigueur leur reconnaît un droit d'usage des ressources dans un cadre réglementé. Le code forestier du Togo autorise l'exercice de droits d'usage strictement limité à la satisfaction des besoins personnels et collectifs des usagers (Titre III, Chapitre $1^{\mathrm{er}}$, Section 2, Article 34).

Les producteurs de bois énergie: Ils sont généralement des professionnels fabricants de charbon de bois et/ou distributeurs de bois de chauffe. Le code forestier interdit l'utilisation de bois vert pour la production de bois énergie. Ce groupe d'acteurs à des intérêts et des objectifs contradictoires avec ceux de l'administration forestière chargée de la mise en application des textes en vigueur. Leurs intérêts peuvent également s'opposer à ceux des organisations non gouvernementales impliquées dans la promotion des ressources forestières et l'environnement durables.

La chefferie traditionnelle : Responsable des us et coutumes, elle veille au respect des interdits et des considérations socioculturelles sur le terroir villageois. Elle est également à l'avant-garde dans la gestion des litiges et conflits inhérents entre autres à l'exploitation des ressources naturelles comme les espèces ligneuses considérées d'ailleurs comme un patrimoine de la communauté. A travers ce rôle, des essences d'espèces ligneuses comme Vitellaria paradoxa, Parkia biglobosa, Vitex doniana, Manguifera indica et Diospyros mespiliformis sont ainsi relativement préservés surtout des coupes systémiques pour la production de bois énergie ou la fabrication de bien artisanal.

Les organismes publics ou gouvernementaux : Ce sont des services de l'administration forestière représentée au niveau central, régional et préfectoral. Dans un rôle régalien, ils sont responsables de la formulation de politiques, de la réglementation du secteur et du contrôle, de la gestion des ressources des parcs, de la délivrance de permis d'exploitation, de la promotion d'une gestion durable, du suivi de l'exploitation et de la traçabilité de celle-ci. Ils ont autorité sur les usagers mais ne peuvent se prévaloir d'une capacité de gestion durable sans l'implication effective des acteurs à la base.

Les chercheurs et spécialistes en gestion des ressources naturelles et de l'environnement : Des chercheurs œuvrent dans les domaines de la biologie, de l'écologie, de la gestion et conservation de la biodiversité et de 
l'aménagement forestier. Engagés dans ces domaines, ils ont pour mission centrale, la formation avancée et la recherche sur tous les aspects liés à la gestion des ressources naturelles et de l'environnement. La force de ce groupe d'acteurs apportant un appui conseil aux décideurs, institutions, organismes publics et autres structures techniques, repose sur l'intégration des savoirs scientifiques. Ainsi, la recherche développe d'une part, des stratégies et des pratiques et participe d'autre part à la formulation de visions, de politiques et de programmes, notamment, par la mise à disposition d'éléments de conception ou d'instruments d'analyse utiles à la prise de décision.

Les organisations non gouvernementales (ONG) : Dans un rôle de donateur ou de partenaire technique pour la promotion d'objectifs sociaux et environnementaux, ces structures de proximité accompagnent les communautés dans la restauration, la régénération et l'enrichissement des espaces boisés. Généralement présentes dans le cadre de projets, elles sont engagées dans des activités d'appui conseil, de communications, de sensibilisation et d'éducation pour la préservation, la gestion durable et la conservation des ressources naturelles. Leurs objectifs très souvent sur un horizon temporel de moyen terme peuvent paraître en contradiction avec ceux des groupes cibles en situation de vulnérabilité exprimant des besoins récurrents de très court terme dont la satisfaction ne peut attendre.

S'inspirant des travaux de Mayers et al., 2005, l'ampleur du défi de préservation des espèces ligneuses est illustrée sur la base d'une estimation de niveaux de potentialité et de pouvoir des parties prenantes identifiées (figure 5). La meilleure situation espérée est celle déterminée par la première bissectrice montrant que certaines parties prenantes doivent être dotées de moyens pour l'apport de contributions positives et d'autres, dépouillées de moyens ou empêchées pour des contributions négatives.

Par rapport aux éleveurs transhumants et généralement pour des intérêts de très court terme, ils peuvent bénéficier de facilités offertes par des propriétaires terriens en violation des règles liées à la délimitation et au respect des couloirs de transhumance. Cependant, étant donné le niveau de leur relation avec les usagers et les risques de conflits qui peuvent en découlés, ces propriétaires n'envisageront pas une relation pérenne avec les transhumants. 


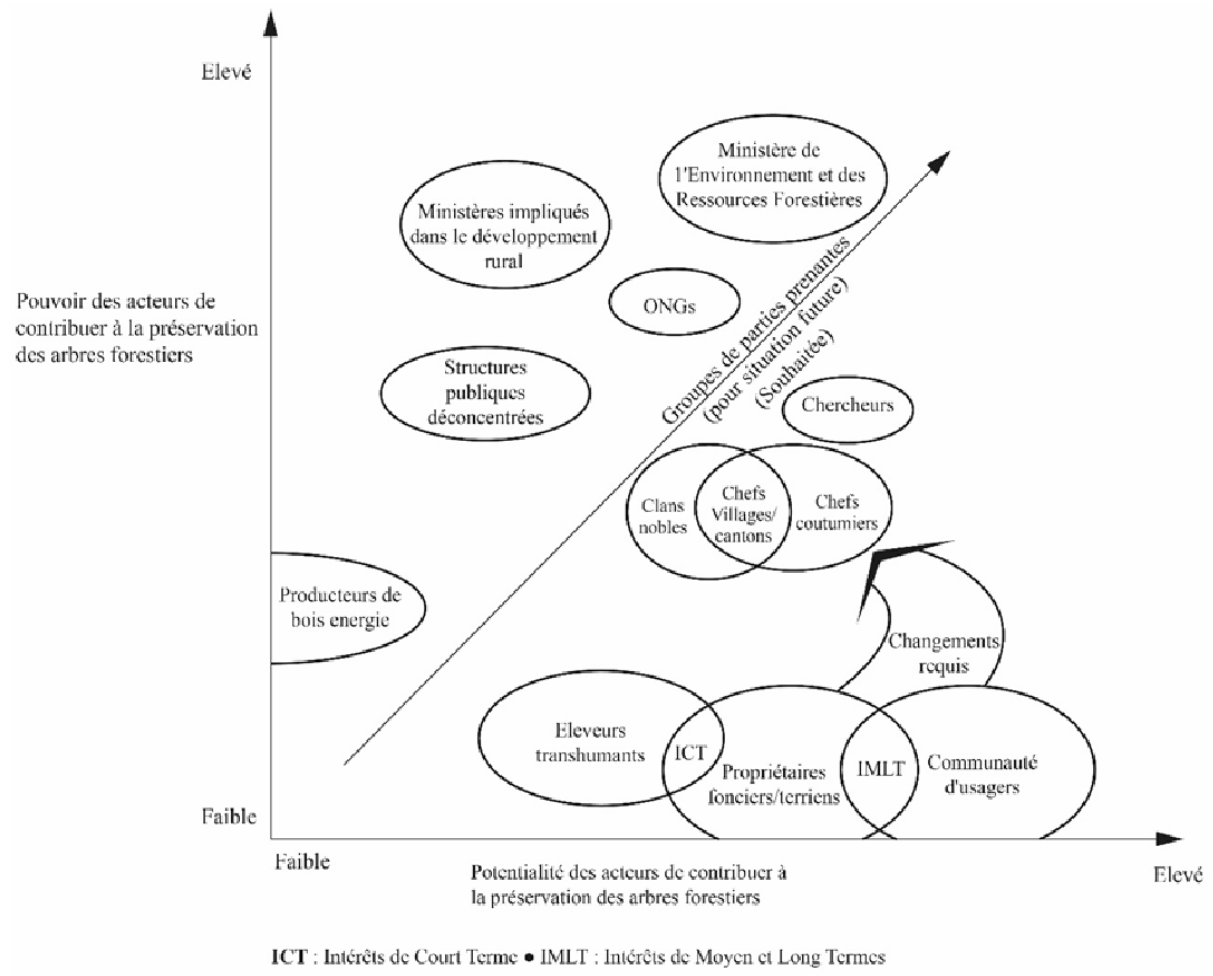

Figure 5 : Groupes des parties prenantes - Potentialité et pouvoir de contribution à la préservation des espèces ligneuses forestières

\section{Discussions}

3.1. Groupes ethnoculturel et domaines d'utilisation des espèces ligneuses des savanes sèches du Nord Togo.

Les groupes socioculturels dans la $\mathrm{s}$ avane sèche se retrouvent, en général, sur le même espace géographique. Ceci confirme qu'en plus des réalités socioculturelles, la localisation géographique du groupe ethnique d'un utilisateur de biens et services éco systémiques, la promiscuité et le contact entre les groupes humains détermineraient les connaissances réelles et les choix de biens et services d'espèces ligneuses utiles du fait du mélange de connaissances culturelles. Ainsi, l'effet explicatif de la tradition ethnique est significatif (Pereki et al., 2012), sous contrainte de disponibilité de l'espèce dans la zone géographique de présence du groupe ethnique. Les résultats de la CCA confirment que les groupes ethniques (G1, G2, G 3, et G4) qui ont des méthodes traditionnelles semblables (Gayibor, 1997) utilisaient pratiquement les mêmes espèces ligneuses pour l'alimentation et pour l'usage médicinal. Au sein des groupes ethniques Konkonba, Lamba, Naouda et Moba par exemple, certaines espèces ligneuses du genre Sterculia et de celui Afzelia ne sont pas utilisées dans la consommation directe. Les raisons évoquées sont les considérations totémiques et magico-spirituelles attribuées à ces espèces 
d'arbres. Ces résultats confirment les conclusions des travaux de Segla et al. (2015). En effet, ces auteurs, en décrivant la relation entre les catégories d'usages faites de Pterocarpus erinaceus et les différentes ethnies ont montré que les Kabyè-Tèm et les Para-Gourma utilisent beaucoup cette dernière comme plante médicinale, bois énergie et fourrage. Allant dans le même sens, Pereki et al. (Pereki et al., 2012) ont indiqué que Sterculia setigera est considéré par les groupes Moba et Konkonba comme « arbre de diables ». Aussi, Afzelia africana est-il considéré par le groupe ethnique Naouda comme un arbre ayant des propriétés magico-spirituelles. Chez les moba, Adansonia digitata, Parkia biglobosa sont utilisés dans les rites spirituels (funéraires).

D'autres espèces comme Pterocarpus erinaceus, Diospyros mespiliformis, Vitex doniana et Borassus aethiopium ont des valeurs artisanales et sont exploitées dans la fabrication de "cache sexe" (utilisé à l'époque dans la savane sèche) et dans la confection de tambours traditionnels. Très clairement, les réalités socioculturelles et la promiscuité entre les groupes ethniques auraient véritablement une incidence sur les connaissances des espèces ligneuses (Rakotoarimanana et al., 2008 ; Atakpama, 2010 ) et les choix opérés par les différents groupes ethniques sur la base entre autres, du « savoir ancien ». Ce savoir est généralement légué par les ascendants et porte surtout sur les connaissances des vertus des plantes servant par exemple d'intrants ou de recettes en médicine traditionnelle (Zohoun, 2002), les valeurs magico-spirituelles de certaines espèces et les totems qu'il convient de respecter au sein des communautés.

De par la multiplicité des usages, la diversité et l'importance avérée des espèces ligneuses utiles dans la vie quotidienne des communautés enquêtées, la présente étude confirme la thèse de Atato et al. (2013), selon laquelle certaines préférences alimentaires relatives à l'utilisation des plantes restent inscrites dans la culture des peuples. La culture alimentaire, la taxonomie populaire et les traditions relatives aux plantes sont d'importants indicateurs de diversité en ce qui concerne les différences de traitement de populations végétales ou d'écotypes d'une même espèce. Les usages des biens et services d'espèces ligneuses permettent aux acteurs impliqués d'interagir directement avec les écosystèmes. C'est le cas des services dits de prélèvement pour la consommation directe ou productive ou encore de services culturels (MEA, 2005).

Partant de la similarité des espèces utilisées par les groupes ethniques ayant des méthodes traditionnelles semblables, les résultats de cette étude montrent que les déterminations de valeurs d'usage tiennent compte des différentes sollicitations d'organes d'espèces ligneuses dont les destinations varient selon les usages connus des populations ciblées. C'est ainsi que certains organes sont plus utilisés que d'autres. Dans la présente étude, les fruits, les feuilles, le tronc (bois), l'écorce et les racines sont les plus utilisés. 
Ces résultats sont confirmés par Segla et al. (2015), puis de Rabiou (2016). Ces auteurs en travaillant sur l'importance socioculturelle, ethnobotanique et perception paysanne de Pterocarpus erinaceus au Togo, Burkina Faso et Niger ont montré eux aussi que le tronc (bois), les feuilles, l'écorce et les racines sont utilisés par environ $100 \%$ des personnes enquêtées. Selon ces mêmes auteurs, les mobiles de prélèvement des différents organes des plantes varient grandement selon les différents groupes socioprofessionnels. Ils affirment par ailleurs que les prélèvements d'organes entraînent des pressions sur les différentes espèces et leurs habitats. Ces différentes pressions anthropiques exercées sur les peuplements ont un impact négatif important, induisant une modification de leur structure et accentuant leur dégradation (Betti, 2001; Barbier, 2007 ; Birregah, 2016 ). Les travaux d' Adjonou et al. (2010) et Duvall (2008) ont montré que les mauvaises pratiques d'exploitation et de prélèvement des organes de la ressource par les différents acteurs (exploitants, bouviers et paysans) impactent le développent et la régénération de l'espèce.

Face à cette situation, l'adoption de mesures visant la conservation et la protection des différentes espèces y compris la gestion des produits forestiers non ligneux sont indispensables pour une utilisation durable de la ressource (Loubelo, 2012).

\subsection{Structuration sociale et relations de pouvoir des parties prenantes dans la $s$ avane sèche}

Au nord Togo, la stratification sociale fait apparaître au sommet, la chefferie traditionnelle qui est en principe, une émanation et une représentation des communautés locales. Ces dernières la reconnaissent et respectent les indications et les orientations de ces personnalités influentes que sont les chefs locaux. Dans certaines localités, notamment chez les peuples Tem ou Kotokoli et Bassar, des chefs de villages ou de cantons sont considérés comme des chefs supérieurs (Alexandre, 1963) et sont assistés dans les prises de décision par des chefs coutumiers issus de différents clans familiaux. Un peu plus au nord chez les peuples Moba, Gourma Gam-Gam et Tchokossi, il apparaît dans la structure sociale, des aînés de clans nobles, crédibles et très respectés. Dans la vie communautaire, ils sont régulièrement consultés par la chefferie et leurs avis comptent aussi bien pour les chefs coutumiers que pour les chefs de villages ou de cantons. Ces derniers dont l'autorité est reconnue dans un espace géographique représentant le village ou le canton sont généralement élus et légalement reconnus par l'administration centrale à travers des arrêtés pris par le Ministre chargé des collectivités locales.

En revanche, les chefs coutumiers qui jouissent d'une grande légitimité et qui semblent avoir l'unanimité de leurs peuples autour de leurs personnes sont désignés dans la majorité des cas sur la base d'une structure lignagère qui 
dépend fondamentalement des structures territoriales et rituelles de chaque milieu. L'influence de ces chefs coutumiers est ainsi mise en évidence et l'analyse des réalités du pouvoir au niveau local permet d'affirmer qu'ils sont véritablement capables de provoquer des changements dans les relations de pouvoirs des parties prenantes.

En définitive, dans l'attribution ou la dévolution de pouvoirs légitime, il ressort que pour une gestion durable des ressources forestières, les chefs coutumiers qui sont rarement influencés par l'administration publique et d'autres forces politiques clivantes ont le potentiel nécessaire pour renforcer les pouvoirs des propriétaires et des usagers terriens dont l'intérêt commun reste la préservation de ce capital naturel (Tisserand, 2011), que sont les espèces ligneuses, source de revenu et de bien-être pour les générations présentes et futures. Ainsi, pour le développement de programmes d'aménagement ou de restauration des forêts, il est important de s'assurer de l'engagement effectif de ces chefs coutumiers à participer à l'émergence des pouvoirs des propriétaires fonciers et de la communauté d'usagers.

\section{Conclusion}

La catégorisation des usages d'espèces identifiées fait ressortir qu'au sein des quatorze groupes ethniques enquêtés et dans un intervalle de significativité de 50 à $75 \%$, les espèces ligneuses exploitées par les communautés ciblées sont généralement utilisées à la fois pour deux ou trois usages et rarement pour un type spécifique d'usage. Par rapport à la similarité des usages, l'ajustement sophistiqué des résultats basé sur l'abondance des espèces d'arbres pour chaque groupe ethnoculturel organise quatre groupes dont deux sous-groupes. En plus des réalités socioculturelles, la localisation géographique du groupe ethnique, la promiscuité et le contact entre les groupes humains déterminent les connaissances réelles et les choix de biens et services d'espèces ligneuses utiles.

Les groupes ethniques qui ont des méthodes traditionnelles semblables utilisent pratiquement d'une part, les mêmes espèces ligneuses pour l'alimentation et l'usage médicinal ou des besoins de fourrage et de pratiques rituelles et d'autre part, pour l'artisanat, le bois énergie et le bois de service mais assez rarement pour un type spécifique d'usage, notamment cosmétique ou hygiénique. Aussi, les parties prenantes ont-elles effectivement une importance, un pouvoir et un potentiel variable pour l'amélioration de la gestion forestière et des biens et services associés. En définitive, au nord du Togo, les espèces ligneuses sont clairement des sources de nourriture pour l'homme et les animaux et pourvoyeurs de nombreux produits médicinaux, hygiéniques et cosmétiques entre autres. Pour la durabilité des écosystèmes forestiers, l'accompagnement des populations devra prendre en compte les considérations socioculturelles des milieux cibles y compris les usages, les 
rôles et responsabilités des parties prenantes, les relations de pouvoirs, les influences et les contradictions d'intérêts ainsi que les préférences des communautés . Ces dernières ont donc des connaissances et attachent des valeurs aux biens et services éco systémiques d'espèces ligneuses. Dans un contexte socioéconomique dynamique, une évaluation contingente permettrait de mesurer le consentement à payer ou le consentement à recevoir des populations pour la préservation des principales espèces forestières plus fréquemment sollicitées dans la s avane sèche au nord Togo.

\section{Remerciements}

Les auteurs adressent leurs sincères remerciements au Groupe de recherche pour le développement durable (GR2D) de l'Université de Lomé pour le cadre de travail scientifique et d'échanges ayant contribué à l'affinement de la problématique de la présente étude. Ils tiennent également à remercier les Directions régionales de l'environnement et des ressources forestières ainsi que les cadres techniques de l'ONG Code Utile Afrique pour leur disponibilité et les apports valeureux dans la collecte des données primaires et secondaires.

\section{References:}

1. Adjonou, K., Ali, N., Kokutse, A. D. \& Kokou, K. (2010). Étude de la dynamique des peuplements naturels de Pterocarpus erinaceus Poir. (Fabaceae) surexploités au Togo. Bois et Forêts des Tropiques, 306 (1).

2. Agboh, N., \& Badjaré, B. (2007). Critères de stratification du Togo en zones homogènes pour la recherche agronomique, Institut Togolais de Recherche Agronomique, rapport d'activités, $64 \mathrm{pp}$.

3. Akpavi, S., Kanda, M., Odah, K., Akpakpah, E. K., Kossi-Titrikou, K., Boutaré, I., \& Akpagana, K. (2013). Valeur socio-culturelle des plantes alimentaires : un facteur de préservation, European scientific journal, (9) 32.

4. Alexandre, P. (1963). Organisation politique des Kotokoli du NordTogo. In: Cahiers d'études africaines, (4) 14, 1963. pp. doi : https://doi.org/10.3406/cea.1963.3719.

5. Aleza, K., Akpagana, K., Atakpama, W., Bayala, J., Dourma, M., Villamor, G.B., \& Wala, K. (2014). Population Structure and Regeneration status of Vitellaria Paradoxa (C. F. Gaertner) under different land management regimes in Atacora department, Benin, Agroforest Syst, 89: 511. doi: 10, 1007/s10457-015-9787-9.

6. Atakpama, W. (2010). Etude des formations à Sterculia setigera Del. dans la zone écofloristique I du Togo: aspects structural et socioéconomiques. DEA de Biologie de développement - Biologie Végétale 
Appliquée. Faculté des Sciences Laboratoire de Botanique et d'Ecologie Végétale, Université de Lomé.

7. Atakpama, W., Batawila, K., Dourma, M., Pereki, H., Wala, K., Dimobe, K., \& Akpagana, K M. Gbeassor, (2012). Ethnobotanical Knowledge of Sterculia setigera Del. in the Sudanian Zone of Togo (West Africa). ISRN Botany.

8. Atakpama, W., Kponor, E.G.E., Kanda, M., Dourma, M., Nare, M., Batawila, K., \& Akpagana K. (2014). Moringa oleifera lamarck (moringaceae) : une ressource phytogénétique à usage multiple, $15 \mathrm{pp}$.

9. Atato, A., Wala, K., Dourma, M., Bellefontaine, R., Woegan, A.Y., Batawila, K., \& Akpagana, K. (2013). Espèces lianescentes à fruits comestibles du Togo. Fruits 67 : 353-368.

10. Badjaré, B., Kokou, K., Bigou-lare, N., Koumantiga, D., Akpakouma, A., Adjayi, M. B. G., \& A. Abbey (2018). Étude ethnobotanique d'espèces ligneuses des savanes sèches au Nord-Togo : diversité, usages, importance et vulnérabilité. BASE.

11. Batawila, K., Akpavi, S., Wala, K., Kanda, M., Vodouhe, R., \& Akpagana, K. (2010). Diversité et gestion des légumes de cueillette au Togo. African Journal of Food Agriculture, Nutrition and Development, ISSN 1684 5374, 21pp.

12. Barbier, S. (2007). Influence de la diversité, de la composition et de l'abondance des essences forestières sur la diversité floristique des forêts tempérées. Ecologie, Environnement. Université d'Orléans.

13. Betti, J. L. (2001). Vulnérabilité des plantes utilisées comme antipaludiques dans l'arrondissement de Mintom au sud de la réserve de biosphère du Dja (Cameroun). National Botanic Garden of Belguim. Systematic and geography of plants, 71 (2): 661 - 678.

14. Birregah, B. W. (2016). Usages endogènes des plantes dans la préfecture de Doufelgou : exploitations, menaces et méthodes de conservation. Université de Lomé, Mémoire master, 98pp.

15. Brahic, E., \& Terreaux, JP. (2009a). Pourquoi et comment estimer la valeur économique de la biodiversité forestière ? Méthodes RDV techniques n 25-26 63-68.

16. Brahic, E., \& Terreaux, JP. (2009b). Evaluation économique de la biodiversité Méthodes et exemple pour les forêts tempérées, Paris France, Edition Quae, 199pp.

17. Dourma, M. (2008). Les forê ts claires a Isoberlinia sp. dans la zone soudanienne du Togo: Ecology, Regeneration naturelle et impacts humains. Thèse de Doctorat, département de Botanique, Université de Lomé, 185 p. 
18. Duvall, C. S. (2008). Pterocarpus erinaceus Poir. In: Louppe, D., Oteng-Amoako, A.A. \& Brink, M. (Editors). Prota 7(1): Timbers/Bois d'œuvre 1, PROTA, Wageningen, Netherlands.

19. Ern, H. (1979). Die Vegetation Togo. Gliederrung, Gefährdung, Erhatung. Willdenowia 9: 295- 31.

20. FAO, (2012). Rapport de l'organisation des Nations Unies pour l'alimentation et l'agriculture sur la situation des forêts.

21. Gayibor, N. L. (1997). Histoire des togolais, des origines à 1884. Presses UB, Lomé, vol. $1: 13$.

22. Guillemette, E. (2014). Caractérisation et suivi des attributs multiressources de la forêt habitée du massif dans un contexte d'aménagement durable, Essai, Université de Laval, Maîtrise en sciences forestières, Maître ès sciences (M. Sc.), 130pp.

23. Hahn-Hadjali, K., Schmidt, M., König, K., Langewiesche, K., Kreft, H., Ouedraogo, A., Agonyissa, D., Thiombiano, A., Agbani, P., Dressler, S., Runge, J., Schareika, N., Sinsin, B., Szarzynski, J., Wittig, R., \& Zizka, G. (2003). Conservation and sustainable use of biodiversity in West Africa-3. A case study: Changes in phytodiversity through human impact. In Sustainable use and conservation of biological diversity- A challenge for society. Proceedings of the international symposium Berlin, PP 154-157.

24. Kebenzikato, A. B., K. Wala, M. Dourma, W. Atakpama, K. Dimobe, H. Pereki, K. Batawila, K. Akpagana (2014). Distribution et structure des parcs à Adansonia digitata L. (baobab) au Togo (Afrique de l'Ouest. African Journals Online, Vol 10, N² (2014), 16pp.

25. Kokou, K., Kokutse, A.D., Aményédzi, A., Adjonou, K., Kpodar, F., \& Guelly, A. K. (2006). Diagnostic socioéconomique sur la valorisation du bambou au Togo. Revue des. Sciences de l'Environnement. Univ. Lomé Togo $2: 527$.

26. Kokou, K., Nuto, Y., Atsri, H. (2009). Impact of charcoal production on woody plant species in West Africa: A case study in Togo. Scientific Research and Essay Vol.4 (9), pp. 881-893.

27. Kremen, C., \& Osfeld, R.S. (2005). A call to ecologists: measuring, analyzing, and managing ecosystem services. Frontiers in Ecology and the Environment, 3(10) : 540-548.

28. Legendre, P., Borcard, D., \& Peres-Neto, P. R. (2005). Analyzing beta diversity: partitioning the spatial variation of community composition data. Ecological Monographs, 75(4), 435-450.

29. Loubelo, E. (2012). Impact des produits forestiers non ligneux (PFNL) sur l'économie des ménages et la sécurité alimentaire : cas de la République du Congo. Economies et finances. Université Rennes 2. $\langle$ NNT : 2012REN20008>. <tel-00713758>. 
30. Luck, G. W., Chan, K., \& Fay, J. (2009). Protecting ecosystem services and biodiversity in the world's watersheds. Conservation Letters, 2, 179-188.

31. Lykke, A. M. (1998). Assessment of species composition change in savanna vegetation by means of woody plants' size class distributions and local information. Biodivers Conserv 7:1261-1275. CrossRefGoogle Scholar.

32. Lykke, A.M., Kristensen, M. K., \& Ganaba S. (2004). Valuation of local use and dynamics of 56 woody species in the Sahel. Biodiversity and Conservation 13:1961-1990, $2004 . \quad$ DOI : 10.1023/B:BIOC.0000035876.39587.1a

33. Mayers, J. (2005). Analyse du pouvoir des parties prenantes, International Institute for Environment and Development (IIED), Power Tools, 28pp.

34. MEA, (2005). Ecosystems and human being. Synthesis 1-24, Millenium Ecosystems Assessment, summary for decision-makers, United Nations, Secretary General Koffi Annan, 25pp.

35. Ouédraogo, I., Nacoulma, B. M. I., Ouédraogo, O., Hahn, K., \& Thiombiano, A. (2014). Productivité et valeur économique des calices de Bombax costatum Pellegr. \& Vuillet en zone soudanienne du Burkina Faso. BOIS \& FORETS DES TROPIQUES, 319(319), 31-41.

36. Pereki, H., Batawila, K., Wala, K., Dourma, M., Akpavi, S., Akpagana, K., Gbeassor, M., \& Ansel, J.L. (2012). Botanical Assessment of Forest Genetic Resources Used in Traditional Cosmetic in Togo (West Africa), Laboratory of Pharmacology-Physiology of Natural Substance, University of Lome.

37. Rabiou, H. (2016). Caractérisation des peuplements naturels de Pterocarpus erinaceus et élaboration de normes de gestion durable au Niger et au Burkina Faso (Afrique de l'Ouest). Thèse de Doctorat, Université de Maradi, Niger, 204 pp.

38. Rakotoarimanana, V., Gondard, H., Ranaivoarivelo, N., \& Carriere, S. (2008). Influence du pâturage sur la diversité floristique, la production et la qualité fourragères d'une savane des Hautes Terres malgaches (région de Fianarantsoa). Sécheresse, 19(1), 39-46.

39. Segla, K.N., Adjonou, K., Rabiou, H., Kamana, P., Radji, A.R., Kokutse, A.D., Bationo, B.A., Mahamane, A. \& Kokou, K. (2015). Importance socio-économique de Pterocarpus erinaceus Poir. au Togo. European Scientific Journal , 11 (23) : 1857-7881.

40. Sop, T. K., Oldeland, J., Schmiedel, U., Ouédraogo, I., \& Thiombiano, A. (2010). Population structure of three woody species in four ethnic domains of the sub-Sahel of Burkina Faso. Land Degradation and Development, DOI 10.1002/ldr. 1026. 
41. Taïta, P. (2003). Use of woody plants by locals in Mare aux Hippotames Biosphere Reserve in Western Burkina Faso. Biodiversity and Conservation, 12: 1205-1217.

42. Traoré, L., Ouédraogo, I., Ouédraogo, A., \& Thiombiano A. (2011). Perceptions, usages et vulnérabilité des ressources végétales ligneuses dans le Sud-Ouest du Burkina Faso. International Journal of Biological and Chemical Sciences, 5(1).

43. Tisserand, F. (2011). Services rendus par les écosystèmes forestiers et scénarios de valorisation au profit des propriétaires, le cas des services liés à l'eau, forêt, nature et société, Agro Paris Tech, 54pp.

44. Wala, K. (2001). Typologie, structure et fonctionnement des agrosystèmes traditionnels dans la préfecture de Doufelgou (NordTogo). Mém. DESS Aménagement et gestion des ressources Naturelles. Univ.Nat. Bénin., F.S.A., 72 p + annexes.

45. Yelkouni, M. (2012). Gestion d'une ressource naturelle et action collective : le cas de la forêt de Tiogo au Burkina Faso. Economies et finances. Thèse unique de doctorat, Université d'Auvergne - ClermontFerrand I, 249pp.

46. Zabouh, K. (2014). Contribution à l'étude des plantes utilisées en tradimédécine vétérinaire dans la région des Savanes du Togo, thèse de doctorat d'Etat en médecine, $76 \mathrm{pp}$.

47. Zohoun, G., Boya, Y., Attolou, M., Adjakidje, V., Oude, P., \& Houndaye, F. (2002). L'utilisation des produits forestiers non ligneux (PFNL) dans le cadre de la gestion forestière durable au Bénin ». Le Flamboyant $\mathrm{n}^{\circ} 55,13-18$. 